Anales del Seminario de Historia de la Filosofía

ISSNe: 1988-2564

http://dx.doi.org/10.5209/ASHF.56809

\title{
Tomás de Vio Cayetano, o la modernidad en letras medievales ${ }^{1}$
}

\author{
Emiliano Javier Cuccia ${ }^{2}$
}

Recibido: 20/03/2017 / Aceptado: 14/06/2017

Resumen. El presente trabajo se propone sintetizar y valorar críticamente los puntos más salientes del recientemente publicado libro Objetividad y ciencia en Cayetano. Una prefiguración de la Modernidad. Como podrá verse, se trata de una obra de gran relevancia para el campo ya que analiza y demuestra el carácter disruptivo que presentan algunas tesis de Tomás de Vio con respecto al pensamiento de Tomás de Aquino, a quien pretende estar comentando. Estos descubrimientos implican importantes consecuencias para el modo de comprender el devenir de la escuela tomística fundada en torno a su legado.

Palabras clave: Tomás de Vio Cayetano; Objetividad; Ciencia; Tomismo; Gnoseología; Metafísica.

\section{[en] Thomas de Vio Cajetan, or the Modernity in Medieval Characters}

\begin{abstract}
This paper aims to summarize and to evaluate the main features of the recently published book Objetividad y ciencia en Cayetano. Una prefiguración de la Modernidad. As it will be clear, it is a work of great importance to the field of the late scholasticism since it analyzes and demonstrates the disruptive character of some of Cajetan's theses with respect to the thought of Thomas Aquinas, whom he asserts to be commenting on. Those findings imply important consequences for the way of understanding the subsequent development of the Thomistic school which was founded around its legacy.
\end{abstract}

Keywords: Thomas de Vio Cajetan; Objectivity; Science; Thomism, Gnoseology; Metaphysics.

Cómo citar: Emiliano Javier Cuccia (2017): "Tomás de Vio Cayetano, o la modernidad en letras medievales", en Anales del Seminario de Historia de la Filosofía 34 (3), 727-734.

La filosofía, como suprema de las ciencias especulativas, posee la particularidad de que el estudio que realiza de su propia historia - lejos de ensimismarla en un círculo autorreferencial y estéril — puede abrirla hacia mayores claridades en su comprensión del mundo. Todo depende de que el mismo se encare con auténtico afán reflexivo y crítico.

Esto es lo que se propone en el estudio publicado recientemente por Ceferino P. D. Muñoz (Universidad de Mendoza, CONICET) y RIL Editores, en colaboración con el Centro de Estudios Tomistas (CET) de la Universidad Santo Tomás de Chile, el cual está basado en la investigación doctoral del académico argentino. El mismo

1 Comentarios al libro de Ceferino p. D. Muñoz Objetividad y ciencia en Cayetano. Una prefiguración de la Modernidad. Ril, Santiago de Chile, 2016.

2 UNCuyo - CONICET.

ecuccia@gmail.com

orcid.org/0000-0003-1401-481X 
fue galardonado con el primer premio de la primera edición del concurso Doctor Humanitatis organizado por el propio CET en el año 2014 y cuenta con un muy interesante prólogo a cargo del doctor Francisco León Florido. Dicho trabajo tiene como objetivo principal el estudio minucioso de los textos del célebre monje dominico —y posteriormente Cardenal — Tomás de Vio Cayetano, quien durante mucho tiempo fuera considerado el más eximio comentador e intérprete del pensamiento de Tomás de Aquino. Con todo, la virtud que distingue la labor de Muñoz es la de encarar un estudio conceptual del pensamiento del originario de Gaeta desde sí mismo, esto es, tomando en cuenta el contexto en que desarrolló sus ideas y el conjunto de influencias recibidas particularmente en su periodo de formación en la Universidad de Padua.

Puntualmente, el foco del análisis está puesto en lo tocante a dos aspectos clave de su obra: el conocimiento intelectual de lo real, y la determinación de los objetos y procedimientos de las ciencias especulativas. Ambos tópicos han sido objeto de numerosos estudios a lo largo de los últimos cien años, aunque entre ellos abundan aquellos que consideran las explicaciones del Cardenal como un desarrollo en continuidad con las ideas de Tomás de Aquino ${ }^{3}$, incluso sin distinguir a veces entre las citas de uno y otro. Naturalmente es sabido que la asumida identidad doctrinal entre ambos autores fue puesta en duda a partir de la segunda mitad del siglo pasado merced a diversos estudios entre los cuales ha resultado paradigmático el de LouisBertrand Geiger sobre el manuscrito autógrafo del comentario al De Trinitate de Boecio $^{4}$, luego profundizado por otros autores ${ }^{5}$. En este caso concreto la disparidad denunciada residiría entre los distintos modos de abstracción intelectual postulados por cada uno de los dominicos y la relación de estos con la distinción de los objetos de ciencia especulativa. Así, la distinción entre una abstracción total y formal o la determinación de tres tipos de ésta última desarrolladas por Cayetano serían aportes originales de éste que no encontrarían un correlato directo en las obras del Aquinate, quien se habría volcado más bien por distinguir entre dos operaciones abstractivas y una separativa.

Muñoz en su libro asume una posición cercana a esta última en tanto admite que los desarrollos de Cayetano presentan diferencias doctrinales y no meramente literales con respecto al Aquinate. Con todo, va claramente más lejos al indicar que existe un hiato radical en los planos de análisis sobre los cuales se maneja cada autor o,

3 Entre ellos puede citarse a Santiago María Ramírez, "De Ipsa Philosophia in Universum Secundum Doctrinam Aristotelico-Thomisticam.” Ciencia Tomista 26 (1922): 33-62; 325-64; Jacques Maritain, Distinguer Pour Unir. Paris,: Desclée, 1932; Leroy, M-V. "Le Savoir Speculatif.” Revue Thomiste: Revue doctrinale de Theologie et de Philosophie 48.1-2 (1948): 236-339; Miguel De Andrea, "Soggetto e oggetto della metafisica secondo S. Tomasso" Angelicum 27 (1950): 165-195; Georges Van Riet, "La Théorie Thomiste De L'abstraction." Revue Philosophique de Louvain (1952): 353-93; Iosephus Grendt, Elementa Philosophiae Aristotelico-thomisticae, Herder, Friburgo-Barcelona, 1953; Simmons, Edward D. "In Defense of Total and Formal Abstraction." New Scholasticism 29 (1955): 427-40 y "The Thomistic Doctrine of the Three Degrees of Formal Abstraction." Thomist: A Speculative Quarterly Review 22 (1959): 37-67

4 Louis Bertrand Geiger, “Abstraction Et Séparation D’après S. Thomas “in De Trinitate' Q.5, A.3.” Revue de sciences philosophiques et théologiques 31 (1947): 3-40

5 Lorenzo Vicente Burgoa, "De Modis Abstractionis Iuxta Sanctum Thomam.” Divus Thomas (1963): 35-65, 189-218, y (1964): 278-99; Philip Merlan, “Abstraction and Metaphysics in St. Thomas' Summa.” Journal of the History of Ideas 14 (1953): 284-91; K. Nordberg, "Abstraction and Separation in the Light of the Historical Roots of Thomas' Tripartition of the Theoretical Sciences." Knowledge and the Sciences in Medieval Philosophy. Proceedings of the Eighth International Congress of Medieval Philosophy (1987). Ed. Tyorinoja, Reijo. Vol. III. Helsinki: Yliopistopaino, 1990. 144-59. 
dicho de un modo más llano, que no sólo existirían diferencias entre las respuestas de cada uno sino que las propias preguntas y problemas que ambos intentan resolver son marcadamente distintas, algo que no siempre ha sido advertido por los especialistas. La causa de esta brecha entre ambos cuerpos doctrinales residiría en la fuerte influencia que el Cardenal recibió de ciertos conceptos provenientes del escotismo al entrar en discusión con los representantes de esta escuela tan en boga en su propio tiempo ${ }^{6}$. De este modo, el intento de contrastar (y contrarrestar) la figura de Escoto con la de Tomás de Aquino - cuya doctrina afirma estar exponiendo fielmente en más de una oportunidad - lo habría llevado a aceptar algunos postulados básicos desarrollados por el escocés o por alguno de sus seguidores a punto tal que el debate quedó planteado en un terreno previamente demarcado por la mens escotista provocando que ciertas ideas clave del pensamiento tomasiano se vean profundamente modificadas al quedar insertas en una cosmovisión que no es la propia.

Precisamente, el análisis del contexto dentro del cual Tomás de Vio desarrolla su clasificación de los tipos de abstracción permite revelar esta discrepancia de base. Dicha clasificación es introducida en su comentario al Proemio del De ente et essentia, lugar donde intenta resolver una objeción de corte escotista: el ente no puede ser lo primero conocido como indica Tomás de Aquino, porque de ser así, entonces la metafísica sería la primera ciencia humana ${ }^{7}$. Ante ello Cayetano responde que el ente puede coronar el acto del entendimiento de tres formas: por abstracción total, formal o neutra. Según este último modo el ente sería lo primero conocido, y sólo merced al acto de abstracción formal, en su nivel más elevado o inmaterial, se constituiría como objeto de la metafísica ${ }^{8}$.

Más allá de la consabida ausencia de una distinción tal en Tomás de Aquino, Ceferino Muñoz desarrolla otros puntos que son clave para mostrar disparidades más profundas entre Cayetano y el Aquinate. En primer lugar, resulta claro que en $\mathrm{Ca}$ yetano el ente como objeto de la metafísica es algo completamente distinto del ente como primero conocido, algo que - según Muñoz - no encuentra antecedentes en el medieval ${ }^{9}$. Pero en segundo lugar, y como fundamento de lo anterior, se deduce que para el de Gaeta aquello que es conocido es un producto del intelecto que depende causalmente de la operación del sujeto para asegurar su formación y constitución. Se trata de una afirmación fuerte en tanto parece ir a contramano del asumido carácter realista de la gnoseología cayetaniana ${ }^{10}$. Por este motivo, y como es esperable, la parte medular del libro está dedicada a su demostración.

6 Cfr. Ceferino Muñoz, Objetividad y ciencia en Cayetano...p. 59.

7 Se trata de una objeción planteada por el escotista Antonio Trombetta, contemporáneo de Cayetano con el que coincidió en la Universidad de Padua. Cfr. Juan Antonio Hevia Echeverría, "Introducción” en Cayetano, Tratado sobre la analogía de los nombres, Tratado sobre el concepto de ente. Pentalfa ediciones, Oviedo, 2005, p. 10 y Ceferino Muñoz, Objetividad y ciencia en Cayetano...p. 88.

8 Cfr. Thomas de Vio Cajetanus, "Super Librum De Ente Et Essentia Sancti Thomae Aquinatis, in Septem Capita Divisus.” Opuscula Omnia. Hildesheim-Zürich-New York: Georg Olms Verlag, 1995. 220-83, pp. 220-223.

9 Cfr. Ceferino Muñoz, Objetividad y ciencia en Cayetano...pp. 81-87. Tal afirmación es refrendada por estudiosos de la talla de Lawrence Dewan. Form and Being. Studies in Thomistic Metaphysics, The Catholic University of America Press, vol. 45, Washington 2006, pp. 35-60.

10 Cabe destacar que Muñoz, para fundamentar esta conclusión, se apoya también en otros estudios muy relevantes como los de Rafael Caldera, La primera captación intelectual. Pamplona, Cuadernos de Anuario Filosófico, 1999; Bernard Besnier, “Cajétan-In 'De Ente et Essentia' quaestio I”, en: B. Pinchard y S. Ricci, (eds.), Rationalisme analogique et humanisme théologique: la culture de Thomas de Vio 'Il Gaetano', Napoles 1993, pp. 71-83; Franco Riva, "Il Gaetano e 1'ente come «primum cognitum»", Rivista di Filosofia Neoescolastica, $85 / 1,1993$, p. 3-20, etc. 
A mi juicio, un punto clave de la mentada demostración consiste en la reseña del modo en que Cayetano comenta un pasaje célebre de la Summa Theologiae. Se trata de la cuestión 85 de la Prima Pars donde el Aquinate explica su posición acerca del modo en que conoce el hombre en estado de vía, refutando el iluminismo agustinista y asumiendo la teoría de corte aristotélico del conocimiento por especies abstraídas desde lo sensible. En el artículo 2 de dicha cuestión se pregunta si tales especies se relacionan con el intelecto como lo que se conoce (quod) o como aquello a través de lo cual se conoce (quo) y Tomás responde que, en líneas generales, la especie es sólo el medio del conocimiento y no lo conocido ${ }^{11}$. Y sólo cuando el intelecto vuelve sobre sí mismo en un acto reflexivo puede decirse que sus especies y conceptos se convierten en lo que se conoce ${ }^{12}$. Pero en su glosa al artículo, Cayetano agrega algunas distinciones por demás relevantes indicando que las especies inteligibles y las concepciones formadas pueden ser consideradas de dos modos:

En un modo, conforme las cosas son cierta [realidad] en el alma: y así ni sobre una ni otra hay conocimiento sino por reflexión. De otro modo, según que las [concepciones] son imágenes o intenciones de las cosas: y así entre ellas hay esta diferencia, porque las especies como imágenes son por las que el intelecto entiende lo que es inteligido [...] Las concepciones en cuanto intenciones son las que [el entendimiento] entiende: y es así porque [...] el concepto presenta la cosa objetivamente. Que las cosas externas sean significadas y sabidas primariamente, y las concepciones no como cosas, sino como intenciones de las cosas externas es lo mismo: porque por el mismo movimiento el alma va a la imagen, y a la cosa, como se dice en De Memoria et Reminiscentia ${ }^{13}$.

Con estas definiciones, el Cardenal estaría introduciendo una diferencia radical con respecto al Aquinate. En efecto, aunque ambos estén distinguiendo entre lo conocido y el medio de conocimiento o - lo que es lo mismo - entre la imagen y el contenido, debe tenerse muy presente que para el segundo este contenido es la propia esencia o naturaleza de la cosa: su quidditas. Esto queda claro por lo manifestado en el propio corpus del artículo: si lo que se conoce no fuese algo extramental sino "una especie presente en el alma, se seguiría que ninguna ciencia trataría sobre las realidades exteriores al alma, sino sólo sobre las especies inteligibles que hay en ella"14 y así toda ciencia se reduciría a la lógica. Pero precisamente, para Cayetano el contenido del conocimiento es una concepción intelectual tomada como intención "que

11 Suma Theologiae I, q. 85, a. 2, s.c.: “...species intelligibilis se habet ad intellectum, sicut species sensibilis ad sensum. Sed species sensibilis non est illud quod sentitur, sed magis id quo sensus sentit. Ergo species intelligibilis non est quod intelligitur actu, sed id quo intelligit intellectus."

12 Cfr. Summa Theologiae I, q. 85, a. 2, co.: "Sed quia intellectus supra seipsum reflectitur, secundum eandem reflexionem intelligit et suum intelligere, et speciem qua intelligit. Et sic species intellectiva secundario est id quod intelligitur. Sed id quod intelligitur primo, est res cuius species intelligibilis est similitude".

13 Tomás de Vio Cajetanus en Thomas Aquinatis. "Pars Prima Summae Theologiae." Opera omnia iussu impensaque Leonis XIII p. M. edita. Roma: Ex Typographia Polyglotta S. C. de Propaganda Fide, 1889. Vol. 5, q. 85, a. 2, XI, p. 335. La traducción y los corchetes pertenecen a Ceferino Muñoz, Objetividad y ciencia en Cayetano..., p. 131 .

14 Summa Theologiae I, q. 85, a. 2, co.: “...eadem sunt quae intelligimus, et de quibus sunt scientiae. Si igitur ea quae intelligimus essent solum species quae sunt in anima, sequeretur quod scientiae omnes non essent de rebus quae sunt extra animam, sed solum de speciebus intelligibilibus quae sunt in anima". 
presenta la cosa objetivamente". Se trata del célebre concepto objetivo que, junto al concepto formal, constituyen dos categorías de análisis muy presentes en el tomismo pero ausentes en el Aquinate ${ }^{15}$. Así, esta modificación lo cambia todo porque, aunque el concepto objetivo tenga un fundamento real, el mismo refleja más bien el modo en que el sujeto cognoscente es afectado por la cosa extramental ${ }^{16}$.

De este modo, más allá de las intenciones que haya albergado Cayetano, su explicación de este pasaje se separa de un modo determinante de la doctrina de Tomás de Aquino al poner un concepto en el lugar de la cosa extramental y desvirtuar así su doctrina gnoseológica. En palabras de Francisco León Florido:

...la noción del ser objetivo parte del presupuesto de que el intelecto no se dirige naturalmente, intencionalmente, hacia la cosa que ha de ser conocida, pues crea su propio objeto de conocimiento que ya no representa la realidad en sí de la cosa tal como es en el mundo, sino la modificación que sufre el intelecto mismo en su $\operatorname{actividad}^{17}$.

Sintetizando, las diferencias que presenta el planteo cayetaniano acerca de la abstracción con respecto a la propuesta de Tomás de Aquino serían para Muñoz mucho más profundas que lo creído hasta ahora en tanto ambos autores se mueven en universos gnoseológicos completamente diversos. Y esta brecha se manifiesta en el modo en que cada uno considera qué es lo conocido por el intelecto: quididad para Tomás, concepto objetivo para Cayetano. Así, una abstracción formal o una total que dé origen a diversos tipos de objetos intelectuales sólo tiene sentido en un universo en el cual "el intelecto y la cosa pertenecen a dos órdenes diferentes"18, y donde lo conocido es algo que debe ser hecho en y por el propio conocimiento. Este universo, que el autor denomina objetivismo, no se corresponde con aquel en que se mueve el Aquinate pero sí con el de Escoto. Irónicamente, al intentar probar contra el Sutil que el ente es lo primero conocido, Cayetano le ha concedido algo mucho más importante: que el ente en tanto conocido es un concepto ${ }^{19}$.

A su vez, la asunción de este contexto objetivista también es común a muchos de los comentaristas y estudiosos de Tomás, tanto clásicos como contemporáneos, e incluso a muchos de aquellos que reconocen hasta cierto punto algunas discrepancias doctrinales entre aquél y Cayetano. El autor cita, por caso, a Leo Elders ${ }^{20}$ pero podrían

15 El autor indica, siguiendo a Tomás Alvira, que estas nociones parecen ser introducidas por ver primera por Juan Capreolo, siendo secundado éste por Soncinas, Cayetano y Juan de Santo Tomas. Cfr. Tomás Alvira, "Esencialismo y verdad" Anuario Filosófico 15, 2 (1982), p. 153. Cit por Ceferino Muñoz, Objetividad y ciencia en Cayetano...p. 128.

16 Autores como Courtine han detectado el papel fundamental que ha tenido el concepto objetivo en el paso a la modernidad filosófica. Cfr. Jean-François Courtine. Il sistema Della metafisica. Tradizione aristotelica e svolta di Suárez. A cura di Constantino Esposito; Prefazione di Giovanni Reale. Vita e Pensiero, Milano, 1999, p. 480.

17 Francisco León Florido, "Una estructura filosófica en Historia de la Filosofía", Anales del Seminario de Historia de la Filosofía, 17 (2000), p. 207. Cit. por Ceferino Muñoz, Objetividad y ciencia en Cayetano...p. 136.

18 Francisco León Florido, "Prólogo" en Ceferino Muñoz, Objetividad y ciencia en Cayetano...p. 16.

19 Quien afirmó esto fue un escotista llamado Juan de Rada que es citado por Muñoz en Objetividad y ciencia en Cayetano...p. 251.

20 Cfr. Leo Elders, Faith and Science: An Introduction to St. Thomas' Expositio in Boethii De Trinitate. Studia Universitatis S Thomae in Urbe. Roma: Herder, 1974; y "La naturaleza de la metafísica según San Alberto Magno y Santo Tomás de Aquino", Scripta Theologica 12, 2 (1980), pp. 557-559. 
sumarse otros autores a la lista como K. Nordberg ${ }^{21}$, P. Merlan ${ }^{22} \mathrm{y}$-más recientemente- R. Pascual ${ }^{23}$ y A. Acerbi ${ }^{24}$. Todos concuerdan, en mayor o menor medida, en que uno de los aportes más notorios de Tomás de Aquino al campo de la gnoseología y la epistemología es el de hacer residir la causa de la distinción de los objetos especulativos (y, por consiguiente, de la tripartición de la ciencia especulativa) en los modos de consideración intelectual - abstractivos o separativo- desarrollados en la cuestión quinta su citado comentario al De Trinitate de Boecio. De este modo, la influencia de la desviación objetivista introducida por Cayetano en la letra del Aquinate parece haberse extendido subrepticiamente entre los estudiosos del segundo como si le fuese cosa propia. Efectivamente, para Muñoz hablar de la operación intelectual abstractiva (o de la separativa, en su defecto) como causa de la constitución de los objetos qua objetos implica comportarse "como si entre el ente real y el ente conocido hubiese un hiato que la inteligencia se ocupa de componer [...] como si hubiera que tender un puente que me asegurara que puedo tener ciencia, y dicha mediación fuese realizada por la abstracción en cuanto inmaterialidad objetiva." ${ }^{25}$

Existen también otras diferencias entre la doctrina de Tomás y las de su comentador explicadas por el autor, que considero interesante rescatar aunque sea brevemente. Una de ellas ya fue introducida más arriba: la consideración meramente conceptual del ente como objeto de la metafísica. Se trataría efectivamente del producto de un grado máximo de abstracción formal que el intelecto formaría al dejar de lado todas las notas que distinguen a los entes entre sí. Pero para Muñoz esto implica un rechazo de dos de las notas más características de la metafísica de Tomás de Aquino: su centralidad en el esse como acto de los actos y su apertura al conocimiento de los entes espirituales. Frente a lo primero, Cayetano parece quedarse en el nivel de un "orden quiditativo o de la esencia" dentro del cual la entitas sería un concepto que representa el aspecto comunísimo a toda esencia y el esse quedaría reducido a la mera existencia fáctica de la $\operatorname{cosa}^{26}$ : un accidente de la esencia ${ }^{27}$. Con respecto a lo segundo, resulta claro que un proceso de abstracción formal no podría alcanzar jamás el conocimiento de lo inmaterial, como lo afirma Tomás con toda claridad en varios lugares ${ }^{28}$.

En las condiciones descriptas, la metafísica se ve transformada en su carácter de ciencia primera y queda limitada a una ontología ${ }^{29}$, es decir un estudio del

${ }^{21}$ K. Nordberg, "Abstraction and Separation in the Light of the Historical Roots of Thomas' Tripartition of the Theoretical Sciences". Knowledge and the Sciences in Medieval Philosophy. Proceedings of the Eighth International Congress of Medieval Philosophy (1987). Ed. Tyorinoja, Reijo. Vol. III. Helsinki: Yliopistopaino, 1990. 144-59

22 Philip Merlan, From Platonism to Neoplatonism. 3 ed. The Hague: Martinus Nijhoff, 1975.

23 Rafael Pascual Aguirre, "La División De Las Ciencias Especulativas En Santo Tomás De Aquino.” Excerpta ex dissertatione ad Doctorandum in Facultate Philosophiae. Pontificia Universitas Gregoriana, 2003.

24 Ariberto Acerbi, “Aquinas's Commentary on Boethius’ De Trinitate.” Review of Metaphysics 66.2 (2012): 317 38.

25 Ceferino Muñoz, Ciencia y objetividad en Cayetano...p. 166.

26 Cfr. Ceferino Muñoz, Ciencia y objetividad en Cayetano...p. 185.

27 Cfr. Ceferino Muñoz, Ciencia y objetividad en Cayetano...p. 104.

28 Cfr. por ejemplo, De veritate, q. 18 a. 5 ad 6, y Summa Theologiae I, q. 88 a. 2 co donde identifica a Avempace como aquel que propuso la posibilidad de que una sucesiva abstracción desmaterializante pudiese permitir el conocimiento de las formas inmateriales subsistentes. Esta misma conclusión ya fue señalada por p. Merlan en "Abstraction and Metaphysics...", p. 287.

29 Esto ya fue advertido por Géry Prouvost, Thomas d'Aquin et les thomistes. Essai sur l'histoire des thomismes, Les Éditions du Cerf, París 1996, pp. 60-65. 
ens cogitabile o conceptual ${ }^{30}$, resignando el ámbito de los seres inmateriales a la teología entendida como una ciencia completamente escindida, al contrario de lo que parece haber indicado Tomás de Aquino ${ }^{31}$. Precisamente, esta escisión cayetaniana entre la metafísica y la teología - y que a la larga termina siendo entre razón y fe ${ }^{32}$ - tendría también raíces en la influencia ejercida en los años de formación del de Gaeta por el llamado aristotelismo paduano que Muñoz define como "la corriente de pensamiento que hace una lectura de Aristóteles desligada del aspecto teológico, y que, en líneas generales, promulga un aristotelismo completamente secular o naturalista" ${ }^{33}$. Según el autor, existen varios elementos que indicarían que Cayetano asume esta lectura de Aristóteles llegando incluso al punto de identificar los límites de la filosofía con los conceptos y temas que el Estagirita trató en sus obras: el padre del Liceo sería el patrón de medida de la disciplina filosófica ${ }^{34}$. Dos casos testigo de esta postura lo constituyen los desarrollos respectivos que el Cardenal hace de las cuestiones sobre si Dios es y sobre la inmortalidad del alma, frente a las cuales se muestra incapaz de resolverlas desde el punto de vista de la metafísica por no encontrar en Aristóteles las nociones filosóficas necesarias ${ }^{35}$.

Ahora bien, tanto la división metafísica-teología recién mentada como la transformación de la primera en una ontología constituyen verdaderos anticipos de lo que serán las características del pensamiento filosófico moderno ${ }^{36}$. A los cuales dos se pueden adicionar por lo menos otros tantos elementos que terminan de perfilar el carácter innovador de la doctrina cayetaniana. El primero de ellos es la caída en un esencialismo que pone en segundo plano la importancia del esse al considerarlo como mero accidente de la esencia, y coloca en esta última toda la razón de la entidad. Esto inclina a "prescindir de la realidad efectiva de las cosas" en tanto la existencia no sería "requerida como actualmente conveniente al objeto en cuanto cognoscible ${ }^{\prime 37}$. Por su parte, el segundo elemento es la prefiguración de la visión moderna del conocimiento como representación de una realidad que no es apta de suyo para ser captada intelectualmente salvo a través de un símil generado por el propio intelecto. Pero, como ya se dijo, esta semejanza - i.e., el concepto objetivono representa tanto la cosa extramental como el modo en que el sujeto es afectado por la misma. Esto implica que "la cosa no comunica su propia forma al sujeto cognoscente - como por ejemplo en Santo Tomás - sino tan solo su representación o imitación" 38 , fruto de la labor conceptualizadora del intelecto.

La eclosión de todas estas características justifican el subtítulo de la obra en cuestión: ciertamente Cayetano se posiciona como un pivote entre el medioevo y la modernidad en materia filosófica al introducir modificaciones a la cosmovisión

\footnotetext{
30 Cfr. Ceferino Muñoz, Ciencia y objetividad en Cayetano...p. 250.

31 Cfr. Ceferino Muñoz, Ciencia y objetividad en Cayetano...p. 197.

32 Cfr. Ceferino Muñoz, Ciencia y objetividad en Cayetano...p. 222.

33 Cfr. Ceferino Muñoz, Ciencia y objetividad en Cayetano...p. 48. Se trataría de un heredero del averroísmo latino que se desarrolló durante buena parte del siglo XIII en la Universidad de París. Muñoz considera como representantes más importantes del aristotelismo paduano a Juan de Jandún y Pedro Pomponazzi.

34 Cfr. Étienne Gilson, “Autour de Pomponazzi”, Archives d' histoire doctrinale et litteraire du Moyen Age, 28 (1961), p. 173, y Ceferino Muñoz, Objetividad y ciencia en Cayetano... p. 221.

35 Cfr. Ceferino Muñoz, Objetividad y ciencia en Cayetano...p. 221.

36 Cfr. Ceferino Muñoz. Objetividad y ciencia en Cayetano...p. 225.

37 Alejandro Llano, Metafísica y lenguaje, Eunsa, Pamplona, 2011, p. 22. Cit. por Ceferino Muñoz, Objetividad y ciencia en Cayetano...p. 227.

38 Ceferino Muñoz, Objetividad y ciencia en Cayetano... p. 241.
} 
medieval pero intentando en todo momento ser fiel al pensamiento de su maestro del siglo XIII y defenderlo en contra de sus rivales escotistas. La demostración detallada y fundamentada de esta situación constituye, a mi juicio, el mayor mérito del trabajo de Ceferino Muñoz.

Sin duda, es posible que el lector extrañe mayores referencias al devenir histórico que separa al Aquinate del Cardenal. En efecto, más allá de lo descripto hasta aquí, las diferencias entre maestro y comentarista no se explican precisamente por un afán rupturista del segundo sino por una extensa y diversa serie de influencias conceptuales que hicieron mella para que la cosmovisión del supuesto comentarista y sus inquietudes intelectuales fueran muy diferentes de las del medieval. Sin embargo resulta necesario tener en cuenta que el objetivo del estudio ha sido otro: no una historia del pensamiento de la segunda escolástica en sus diversas ramas, sino el análisis de la mens cayetaniana y la explicitación de sus diferencias de plano con el universo conceptual de Tomás de Aquino, al menos en los aspectos mencionados más arriba.

En definitiva, Objetividad y ciencia en Cayetano constituye el resultado de una investigación que pone el foco en un periodo poco estudiado por la historiografía filosófica, y en una figura que - en líneas generales - ha sido tenida por secundaria. Pero esto lo hace desde una óptica no historiográfica sino más bien conceptual, al analizar el peso filosófico de la diferencia de planos analíticos entre Tomás y el de Gaeta, teniendo en cuenta la intención explícita del propio Cardenal de ser en todo momento fiel al pensamiento del Aquinate. Con todo, este estudio ha sido llevado adelante con tal riqueza en las fuentes citadas y cuidado en la reflexión que el lector se verá estimulado a indagar con mayor profundidad en un auténtico mundo conceptual que verá abierto ante sí y cuya importancia descubrirá radical para la comprensión del desarrollo de la filosofía en Occidente. 